\title{
A Study on Two Types of Multiple Integrals
}

\author{
Chii-Huei Yu \\ Department of Management and Information, Nan Jeon University of Science and Technology, Tainan City, 73746, Taiwan \\ *Corresponding Author: chiihuei@mail.njtc.edu.tw
}

Copyright (C) 2014 Horizon Research Publishing All rights reserved.

\begin{abstract}
This paper takes the mathematical software Maple as the auxiliary tool to study two types of multiple integrals. We can obtain the closed forms of these two types of multiple integrals. On the other hand, we propose two examples to do calculation practically. The research methods adopted in this study involved finding solutions through manual calculations and verifying these solutions by using Maple. This type of research method not only allows the discovery of calculation errors, but also helps modify the original directions of thinking from manual and Maple calculations. For this reason, Maple provides insights and guidance regarding problem-solving methods.
\end{abstract}

Keywords Multiple Integrals, Closed Forms, Maple

\section{Introduction}

The multiple integral problem is closely related with probability theory and quantum field theory, and can refer to [1-2]. For this reason, the evaluation and numerical calculation of multiple integrals is important. In this study, we evaluate the following two types of $n$-tuple integrals

$$
\begin{aligned}
& \int_{r_{n}}^{s_{n}} \cdots \int_{r_{1}}^{s_{1}} \exp \left(\sum_{k=1}^{n} a_{k} x_{k}\right) \cos \left(\sum_{k=1}^{n} b_{k} x_{k}+c\right) d x_{1} \cdots d x_{n} \\
& \int_{r_{n}}^{s_{n}} \cdots \int_{r_{1}}^{s_{1}} \exp \left(\sum_{k=1}^{n} a_{k} x_{k}\right) \sin \left(\sum_{k=1}^{n} b_{k} x_{k}+c\right) d x_{1} \cdots d x_{n}
\end{aligned}
$$

Where $n$ is any positive integer, $c, a_{k}, b_{k}, r_{k}, s_{k}$ are real numbers, $a_{k}^{2}+b_{k}^{2} \neq 0$ for $k=1, . ., n$. We can obtain the closed forms of these two types of multiple integrals; these are the major results of this study (i.e., Theorems 1 and 2). Moreover, we obtain some corollaries from these two theorems. For the study of related multiple integral problems can refer to [3-11]. In addition, we provide some multiple integrals to do calculation practically. The research methods adopted in this study involved finding solutions through manual calculations and verifying these solutions by using Maple. This type of research method not only allows the discovery of calculation errors, but also helps modify the original directions of thinking from manual and Maple calculations. Therefore, Maple provides insights and guidance regarding problem-solving methods.

\section{Main Results}

Firstly, we introduce some notations and some properties used in this paper.

\subsection{Notations}

2.1.1. Let $z=a+i b \in C$, where $i=\sqrt{-1}, a, b$ are real numbers, and $C$ is the set of all complex numbers. We denote $a$ the real part of $z$ by $\operatorname{Re}(z)$, and $b$ the imaginary part of $z$ by $\operatorname{Im}(z)$.

2.1.2. $\prod_{k=1}^{n} p_{k}=p_{1} p_{2} \cdots p_{n}$, where $p_{k} \in C$ for all $k=1, . ., n$.

\subsection{Euler's formula ([12])}

$e^{i x}=\cos x+i \sin x$, where $x$ is any real number.

\subsection{Fundamental theorem of calculus ([13])}

Let $G$ be open in $C$, and let $\gamma$ be a rectifiable path in $G$ with initial and end points $\alpha$ and $\beta$ respectively. If $f: G \rightarrow C$ is a continuous function with a primitive $F: G \rightarrow C$, then $\int_{\gamma} f=F(\beta)-F(\alpha)$.

( $F$ is a primitive of $f$ if $\frac{d}{d z} F(z)=f(z)$.)

Before deriving the major results in this study, we need a lemma.

\subsection{Lemma A}

Suppose $a, b, r, s$ are real numbers, $a^{2}+b^{2} \neq 0$. Then the integral 


$$
\int_{r}^{s} \exp [(a+i b) x] d x=\frac{\exp [(a+i b) s]-\exp [(a+i b) r]}{a+i b}
$$

\subsubsection{Proof}

Because $\frac{d}{d z}\left[\frac{1}{\lambda} \exp (\lambda z)\right]=\exp (\lambda z)$,

where $\lambda, z \in C, \lambda=a+i b \neq 0$. Let $\gamma$ be a line in $x$-axis with initial and end points $r$ and $s$ respectively.

Then we have

$$
\begin{gathered}
\int_{r}^{s} \exp [(a+i b) x] d x \\
=\int_{\gamma} \exp (\lambda z) d z \\
=\frac{1}{\lambda}[\exp (\lambda s)-\exp (\lambda r)] \quad(\text { Using } \\
=\frac{\exp [(a+i b) s]-\exp [(a+i b) r]}{a+i b}
\end{gathered}
$$

The following is the first major result in this study, we determine the closed forms of the multiple integral (1).

\subsection{Theorem 1}

If $n$ is any positive integer, and $c, a_{k}, b_{k}, r_{k}, s_{k}$ are real numbers, $a_{k}^{2}+b_{k}^{2} \neq 0$ for $k=1, . ., n$. Then the $n$ -tuple integral

$$
\begin{aligned}
& \int_{r_{n}}^{s_{n}} \cdots \int_{r_{1}}^{s_{1}} \exp \left(\sum_{k=1}^{n} a_{k} x_{k}\right) \cos \left(\sum_{k=1}^{n} b_{k} x_{k}+c\right) d x_{1} \cdots d x_{n} \\
& =\operatorname{Re}\left[\exp (i c) \cdot \prod_{k=1}^{n} \frac{\exp \left[\left(a_{k}+i b_{k}\right) s_{k}\right]-\exp \left[\left(a_{k}+i b_{k}\right) r_{k}\right]}{a_{k}+i b_{k}}\right]
\end{aligned}
$$

\subsubsection{Proof}

$\int_{r_{n}}^{s_{n}} \cdots \int_{r_{1}}^{s_{1}} \exp \left(\sum_{k=1}^{n} a_{k} x_{k}\right) \cos \left(\sum_{k=1}^{n} b_{k} x_{k}+c\right) d x_{1} \cdots d x_{n}$ $=\int_{r_{n}}^{s_{n}} \cdots \int_{r_{1}}^{s_{1}} \operatorname{Re}\left[\exp \left(\sum_{k=1}^{n} a_{k} x_{k}\right) \exp i\left(\sum_{k=1}^{n} b_{k} x_{k}+c\right)\right] d x_{1} \cdots d x_{n}$

(By Euler's formula)

$=\operatorname{Re}\left[\exp (i c) \cdot \int_{r_{n}}^{s_{n}} \cdots \int_{r_{1}}^{s_{1}} \exp \left(\sum_{k=1}^{n}\left(a_{k}+i b_{k}\right) x_{k}\right) d x_{1} \cdots d x_{n}\right]$

$$
=\operatorname{Re}\left[\exp (i c) \cdot \prod_{k=1}^{n} \int_{r_{k}}^{s_{k}} \exp \left[\left(a_{k}+i b_{k}\right) x_{k}\right] d x_{k}\right]
$$

$=\operatorname{Re}\left[\exp (i c) \cdot \prod_{k=1}^{n} \frac{\exp \left[\left(a_{k}+i b_{k}\right) s_{k}\right]-\exp \left[\left(a_{k}+i b_{k}\right) r_{k}\right]}{a_{k}+i b_{k}}\right]$

$$
\text { (By Lemma A) }
$$

By Theorem1, we immediately have the following result.

\subsection{Corollary 1}

Suppose $n$ is any positive integer, $c, a_{k}, b_{k}$ are real numbers, $a_{k}<0$ for $k=1, . ., n$. Then the $n$-tuple improper integral

$$
\begin{gathered}
\int_{0}^{\infty} \cdots \int_{0}^{\infty} \exp \left(\sum_{k=1}^{n} a_{k} x_{k}\right) \cos \left(\sum_{k=1}^{n} b_{k} x_{k}+c\right) d x_{1} \cdots d x_{n} \\
=(-1)^{n} \cdot \operatorname{Re}\left[\frac{\exp (i c)}{\prod_{k=1}^{n}\left(a_{k}+i b_{k}\right)}\right]
\end{gathered}
$$

In Corollary 1, taking $x_{k}=\ln y_{k}$, where $y_{k} \geq 1$ for $k=1, . ., n$. Then we obtain the following result.

\subsection{Corollary 2}

If the assumptions are the same as Corollary 1 , then the $n$ -tuple improper integral

$$
\begin{gathered}
\int_{1}^{\infty} \cdots \int_{1}^{\infty} \prod_{k=1}^{n} y_{k}{ }^{a}{ }^{-1} \cdot \cos \left(\sum_{k=1}^{n} b_{k} \ln y_{k}+c\right) d y_{1} \cdots d y_{n} \\
=(-1)^{n} \cdot \operatorname{Re}\left[\frac{\exp (i c)}{\prod_{k=1}^{n}\left(a_{k}+i b_{k}\right)}\right]
\end{gathered}
$$

The following is the second major result in this paper, we obtain the closed forms of the multiple integral (2).

\subsection{Theorem 2}

If the assumptions are the same as Theorem 1. Then the $n$ -tuple integral

$$
\begin{aligned}
& \int_{r_{n}}^{s_{n}} \cdots \int_{r_{1}}^{s_{1}} \exp \left(\sum_{k=1}^{n} a_{k} x_{k}\right) \sin \left(\sum_{k=1}^{n} b_{k} x_{k}+c\right) d x_{1} \cdots d x_{n} \\
& =\operatorname{Im}\left[\exp (i c) \cdot \prod_{k=1}^{n} \frac{\exp \left[\left(a_{k}+i b_{k}\right) s_{k}\right]-\exp \left[\left(a_{k}+i b_{k}\right) r_{k}\right]}{a_{k}+i b_{k}}\right]
\end{aligned}
$$

\subsubsection{Proof}

$\int_{r_{n}}^{s_{n}} \cdots \int_{r_{1}}^{s_{1}} \exp \left(\sum_{k=1}^{n} a_{k} x_{k}\right) \sin \left(\sum_{k=1}^{n} b_{k} x_{k}+c\right) d x_{1} \cdots d x_{n}$ $=\int_{r_{n}}^{s_{n}} \cdots \int_{\eta_{1}}^{s_{1}} \operatorname{Im}\left[\exp \left(\sum_{k=1}^{n} a_{k} x_{k}\right) \exp i\left(\sum_{k=1}^{n} b_{k} x_{k}+c\right)\right] d x_{1} \cdots d x_{n}$ (By Euler's formula) 


$$
\begin{array}{r}
=\operatorname{Im}\left[\exp (i c) \cdot \prod_{k=1}^{n} \int_{r_{k}}^{s_{k}} \exp \left[\left(a_{k}+i b_{k}\right) x_{k}\right] d x_{k}\right] \\
=\operatorname{Im}\left[\exp (i c) \cdot \prod_{k=1}^{n} \frac{\exp \left[\left(a_{k}+i b_{k}\right) s_{k}\right]-\exp \left[\left(a_{k}+i b_{k}\right) r_{k}\right]}{a_{k}+i b_{k}}\right]
\end{array}
$$

(By Lemma A)

Using Theorem 2, we have the following result.

\subsection{Corollary 3}

If the assumptions are the same as Corollary 1 , then the $n$ -tuple improper integral

$$
\begin{aligned}
& \int_{0}^{\infty} \cdots \int_{0}^{\infty} \exp \left(\sum_{k=1}^{n} a_{k} x_{k}\right) \sin \left(\sum_{k=1}^{n} b_{k} x_{k}+c\right) d x_{1} \cdots d x_{n} \\
& =(-1)^{n} \cdot \operatorname{Im}\left[\frac{\exp (i c)}{\prod_{k=1}^{n}\left(a_{k}+i b_{k}\right)}\right]
\end{aligned}
$$

In Corollary 3 , let $x_{k}=\ln y_{k}$, where $y_{k} \geq 1$ for $k=1, . ., n$. Then the following result holds.

\subsection{Corollary 4}

If the assumptions are the same as Corollary 1 , then the $n$ -tuple improper integral

$$
\begin{aligned}
& \int_{1}^{\infty} \cdots \int_{1}^{\infty} \prod_{k=1}^{n} y_{k}^{a_{k}-1} \cdot \sin \left(\sum_{k=1}^{n} b_{k} \ln y_{k}+c\right) d y_{1} \cdots d y_{n} \\
& =(-1)^{n} \cdot \operatorname{Im}\left[\frac{\exp (i c)}{\prod_{k=1}^{n}\left(a_{k}+i b_{k}\right)}\right]
\end{aligned}
$$

\section{Examples}

In the following, for the two types of multiple integrals in this study, we provide two examples and use Theorems 1, 2 and Corollaries 1, 2, 3, 4 to determine the closed forms of these multiple integrals. On the other hand, we employ Maple to calculate the approximations of these multiple integrals and their solutions for verifying our answers.

3.1. Example 1 By Theorem 1, we obtain the double integral

$$
\int_{\pi / 2}^{2 \pi} \int_{\pi / 3}^{\pi} \exp \left(2 x_{1}-3 x_{2}\right) \cos \left(5 x_{1}+4 x_{2}+\frac{\pi}{3}\right) d x_{1} d x_{2}
$$

$$
\begin{array}{r}
=\operatorname{Re}\left[\frac{\exp \left(i \frac{\pi}{3}\right) \cdot\left[\exp \pi(2+5 i)-\exp \frac{\pi}{3}(2+5 i)\right]}{(2+5 i)(-3+4 i)} \times\right. \\
\left.\left[\exp 2 \pi(-3+4 i)-\exp \frac{\pi}{2}(-3+4 i)\right]\right]
\end{array}
$$

$$
=\frac{(26+7 \sqrt{3})\left[\exp (-4 \pi)-\exp \frac{\pi}{2}\right]+52\left[\exp \left(\frac{-16 \pi}{3}\right)-\exp \left(\frac{-5 \pi}{6}\right)\right]}{1450}
$$

We use Maple to verify the correctness of (10) as follows:

$>$ evalf(Doubleint $(\exp (2 * x 1-3 * x 2) * \cos (5 * x 1+4 * x 2+\mathrm{Pi} / 3)$, $\mathrm{x} 1=\mathrm{Pi} / 3 . . \mathrm{Pi}, \mathrm{x} 2=\mathrm{Pi} / 2 . .2 * \mathrm{Pi}), 14)$;

\section{$-0.129096252409693097$}

$>\operatorname{evalf}(((26+7 * \operatorname{sqrt}(3)) *(\exp (-4 * \mathrm{Pi})-\exp (\mathrm{Pi} / 2))+52 *(\exp (-16$ $* \mathrm{Pi} / 3)-\exp (-5 * \mathrm{Pi} / 6))) / 1450,18)$;

\section{$-0.129096252409693097$}

Also, by Corollary 1, we obtain the triple improper integral $\int_{0}^{\infty} \int_{0}^{\infty} \int_{0}^{\infty} \exp \left(-6 x_{1}-x_{2}-2 x_{3}\right) \cos \left(x_{1}+x_{2}+4 x_{3}-\frac{\pi}{4}\right) d x_{1} d x_{2} d x_{3}$

$$
\begin{gathered}
=-\operatorname{Re}\left[\frac{\exp \left(-i \frac{\pi}{4}\right)}{(-6+i)(-1+i)(-2+4 i)}\right] \\
=\frac{\sqrt{2}}{185} \approx 0.0076443976344491
\end{gathered}
$$

On the other hand, using Corollary 2, the following double improper integral

$$
\begin{gathered}
\int_{1}^{\infty} \int_{1}^{\infty} y_{1}{ }^{-2} y_{2}{ }^{-4} \cdot \cos \left(5 \ln y_{1}-4 \ln y_{2}+\frac{\pi}{3}\right) d y_{1} d y_{2} \\
=\operatorname{Re}\left[\frac{\exp \left(i \frac{\pi}{3}\right)}{(-1+5 i)(-3-4 i)}\right] \\
=\frac{23-11 \sqrt{3}}{1300} \approx 0.003036491623
\end{gathered}
$$

\subsection{Example 2}

By Theorem 2, the double integral

$\int_{\pi / 2}^{\pi} \int_{\pi / 4}^{2 \pi} \exp \left(4 x_{1}+2 x_{2}\right) \sin \left(8 x_{1}-4 x_{2}+\frac{3 \pi}{4}\right) d x_{1} d x_{2}$ $=\operatorname{Im}\left[\frac{\exp \left(i \frac{3 \pi}{4}\right) \cdot\left[\exp 2 \pi(4+8 i)-\exp \frac{\pi}{4}(4+8 i)\right]}{(4+8 i)(2-4 i)} \times\right.$ 


$$
\begin{gathered}
\left.\left[\exp \pi(2-4 i)-\exp \frac{\pi}{2}(2-4 i)\right]\right] \\
=\frac{\sqrt{2}(\exp 8 \pi-\exp \pi)(\exp 2 \pi-\exp \pi)}{80} \\
\approx 7.44737801177467992 \times 10^{11}
\end{gathered}
$$

Also, by Corollary 3 , the triple improper integral

$$
\begin{gathered}
\int_{0}^{\infty} \int_{0}^{\infty} \int_{0}^{\infty} \exp \left(-x_{1}-3 x_{2}-x_{3}\right) \sin \left(2 x_{1}-x_{2}+3 x_{3}+\frac{\pi}{6}\right) d x_{1} d x_{2} d x_{3} \\
=-\operatorname{Im}\left[\frac{\exp \left(i \frac{\pi}{6}\right)}{(-1+2 i)(-3-i)(-1+3 i)}\right] \\
=\frac{2 \sqrt{3}-1}{100} \approx 0.024641016151378
\end{gathered}
$$

By Corollary 4, we get the double improper integral

$$
\begin{array}{r}
\int_{1}^{\infty} \int_{1}^{\infty} y_{1}^{-4} y_{2}^{-3} \cdot \sin \left(7 \ln y_{1}+2 \ln y_{2}-\frac{3 \pi}{4}\right) d y_{1} d y_{2} \\
=\operatorname{Im}\left[\frac{\exp \left(-i \frac{3 \pi}{4}\right)}{(-3+7 i)(-2+2 i)}\right] \\
=\frac{-3 \sqrt{2}}{232} \approx-0.01828724439151
\end{array}
$$

\section{Conclusion}

As mentioned, the multiple integral problem is important in probability theory and quantum field theory. In this study, we propose a new technique to solve some types of multiple integrals, and we hope this method can be applied in mathematical statistics or quantum physics. On the other hand, Maple also plays a vital assistive role in problem-solving. In the future, we will extend the research topic to other calculus and engineering mathematics problems and solve these problems by using Maple. These results will be used as teaching materials for Maple on education and research to enhance the connotations of calculus and engineering mathematics.

\section{REFERENCES}

[1] F. Streit, On multiple integral geometric integrals and their applications to probability theory, Canadian Journal of Mathematics, Vol. 22, pp. 151-163, 1970.

[2] L. H. Ryder, Quantum Field Theory, 2nd ed., New York: Cambridge University Press, 1996.

[3] C. -H. Yu, A study on the multiple improper integral problems, Journal of Hsin Sheng, in press.

[4] C. -H. Yu, Using Maple to study multiple improper integrals, International Journal of Research in Information Technology, Vol. 1, Issue. 8, pp. 10-14, 2013.

[5] C.-H. Yu, A study on the multiple improper integral problems with Maple, Applied Mechanics and Materials, in press.

[6] C. -H. Yu, Application of Maple on evaluating multiple improper integrals, Proceedings of the 6th IEEE/International Conference on Advanced Infocomm Technology, Taiwan, No. 00282, 2013.

[7] C. - H. Yu, Using Maple to study the multiple improper integral problem, Proceedings of IIE Asian Conference 2013, Taiwan, Vol. 1, pp. 625-632, 2013.

[8] C. -H. Yu, Application of Maple: the evaluation of double integral as an example, Proceedings of 2013 International Symposium on intercultural communication, Taiwan, pp. 294-302, 2013.

[9] C. -H. Yu, Application of Maple on the evaluation of four types of double integrals, Proceedings of 2013 Business Innovation and Development Conference, Taiwan, B20130117002, 2013.

[10] C. -H. Yu, Application of Maple on solving some type of multiple integrals, Proceedings of the 8th Information Management and Practice Conference, Taiwan, TP20120128, 2012.

[11] C. -H. Yu, Application of Maple: multiple integrals as examples, Proceedings of the 6th Cross-Strait Technology and Humanities Education and University-Industry Cooperation Seminar, Taiwan, EMD001, 2012.

[12] R. Courant and F. John, Introduction to Calculus and Analysis, Vol. 1, New York: Springer-Verlag, p551, 1989.

[13] J. B. Conway, Functions of One Complex Variable, 2nd edition, Graduate Texts in Mathematics, New York: Springer-Verlag, p65, 1978. 